\title{
Actualités sur les douleurs cranio-faciales
}

\author{
Dupui P \\ Service d'Explorations fonctionnelles physiologiques, CHU Rangueil, Toulouse, France \\ dupui@cict.fr
}

Les douleurs crânio-faciales sont une cause fréquente de consultation chez le généraliste et le neurologue; elles concernent aussi le chirurgien dentiste car certaines d'entre-elles sont dues à une pathologie de la sphère stomatognathique.

Le premier type de douleurs correspond à celles qui apparaissent spontanément dans la région céphalique. Elles sont parmi les plus pénibles et ont des aspects multiples. Parmi les plus fréquentes d'entre-elles, on retrouve les douleurs dentaires qui ont, le plus souvent, pour origine une inflammation de la pulpe. Ces douleurs disparaissent généralement lorsqu'on supprime les stimulations périphériques qui les provoquent. Ces douleurs, heureusement transitoires, répondent bien aux antalgiques.

Le deuxième type de douleurs céphaliques correspond aux céphalées chroniques et aux douleurs de longue durée. C'est le cas par exemple des :

- douleurs qui apparaissent lors d'un zona de la face. Elles sont dues à une perte partielle des afférences, le zona supprimant la conduction nerveuse dans les fibres de grand diamètre provenant d'une région périphérique localisée,

- douleurs accompagnant une désafférentation totale ou partielle d'une région cutanée de la face, de la muqueuse buccale et parfois la perte d'une dent. Elles rappellent celles observées après l'amputation d'un membre. Elles sont produites par des lésions sur le trajet des afférences trigéminales,

- douleurs de la migraine qui peuvent être accompagnées de troubles sensoriels (visuels...) et/ou moteurs dont l'étiopathogénie (à la fois neurogène et vasculaire) ; la migraine correspond à une diminution plus ou moins étendue du débit sanguin dans certaines zones focalisées du cortex cérébral,

- douleurs des tics douloureux de la face ou névralgie essentielle du trijumeau dues à l'apparition d'un foyer irritatif dans les structures centrales de projection des afférences de la face.

La reconnaissance et la prise en charge de ces différentes douleurs chroniques posent problème et nécessitent une bonne connaissance des processus physiopathogéniques. Ces douleurs chroniques répondent peu ou pas du tout aux antalgiques classiques. Les différents moyens spécifiques de prise en charge seront développés lors de l'exposé oral. 